\title{
Heavy quarkonium hybrids from Coulomb gauge QCD
}

\author{
Peng Guo and Adam P. Szczepaniak \\ Physics Department and Nuclear Theory Center \\ Indiana University, Bloomington, IN 47405, USA. \\ Giuseppe Galatà, Andrea Vassallo and Elena Santopinto \\ I.N.F.N. and Dipartimento di Fisica, \\ via Dodecaneso 33, Genova, I-16146, Italy.
}

(Dated: March 1, 2022)

\begin{abstract}
Using the non-relativisitc reduction of Coulomb gauge QCD we compute spectrum of the low mass hybrid mesons containing a heavy quark-antiquark pair. The gluon degrees of freedom are treated in the mean field approximation calibrated to the gluelump spectrum. We discuss the role of the non-abelian nature of the QCD Coulomb interaction in the ordering of the spin-parity levels.
\end{abstract}

PACS numbers: 11.10Ef, 12.38.Mk, 12.40.-y, 12.38.Lg

Keywords:

\section{INTRODUCTION}

Gluons are responsible for almost the entire mass of light hadrons and contribute significantly to hadron spin. Gluons have been probed in hard processes while spectroscopy of gluonic excitations has so far been very limited to a few potential hybrid and glueball candidates. In the near future, however experiments at JLab, PANDA and BESIII are expected to significantly advance our knowledge of gluonic excitations through measurements of decay channels that couple to exotic quantum numbers carried by hybrids. PANDA and BESIII will in particular be sensitive to charmonium hybrids.

Modern studies of gluon dominated hadron spectra have been possible through lattice simulations [1-3] while in early days of hadron phenomenology various effective models have been developed [4-11]. Among these the constituent, or quasi-particle approach have had may successes in particular in the context of heavy quarkonia. For gluonic excitations in presence of heavy quarks the Born-Oppenheimer approximation can be employed to replace the (fast) gluon field by an effective potential between the non-relativistic quarks [12]. These effective potentials originate from excited gluon configurations and can be reliably computed using lattice gauge simulations and afterwards used to construct hybrid heavy quarkonia [13]. Keeping the quark-antiquark sources static and taking their separation to zero results in a spectrum of the so called gluelumps which describe the gluon field bound to a static color octet source [2]. It is thus natural to expect that there should be a close relation between spectrum of gluelumps and heavy quarkonium hybrids. This is because in the latter the average spacial separation between heavy quarks is small, of the order of $\langle r\rangle \sim O\left(1 /\left(\alpha_{s} m\right)\right)$ where, $m$ is the heavy quark mass and $\alpha_{s}$ is the running coupling evaluated at distance scale $O(\langle r\rangle)$.

Recent lattice simulations of the charmonium spectrum in quenched QCD beyond the Born-Oppenheimer approximation also lead to more states then predicted by the quarkonium potential models potentially indicating presence of gluonic excitations [14]. In the quenched approximation and with non-relativistic quarks the connection between heavy quarkonium spectrum and the potential model can be made precise. In the context of the Wilson loop formalism this has been done in [15-17] where potential matrix elements between heavy quarkonium states were related to correlation functions containing QCD gluon field operators. In [18] the FoldyWouthousen formalism was used instead and the effective non-relativistic QCD Hamiltonian in the Coulomb gauge was derived. In this framework all operators conserve the heavy quark quark number and depend on the gluon field. The effective $Q \bar{Q}$ potential matrix elements in general depend on the distribution of the gluon field in the system. In general, gluon distribution in the vacuum and in a state containing a $Q \bar{Q}$ pair are different. In [19] it was found that at large separations the Coulomb energy exceeds that of true static quarkonium state. This makes possible to interpret the former as a variational approximation to the true $Q \bar{Q}$ QCD state. This difference has simple interpretation in the Coulomb gauge [20, 21]. The Coulomb energy corresponds to the energy of an approximate, variational state with the $Q \bar{Q}$ pair added to the QCD vacuum. Gluon-number changing interactions in the Coulomb potential couple the $Q \bar{Q}$ source to an arbitrary number of transverse gluons which eventually produces the string-like QCD $Q \bar{Q}$ eigenstate whose energy is that of the Wilson loop. Extrapolating to small $Q \bar{Q}$ separations, however, a reasonable initial approximation would be to assume that the gluon field is not significantly disturbed by the heavy quark sources and to build the spectrum of gluon excitation using an effective gluon Fock space that is independent from the sources. Such a spectrum was constructed in [22-28] using a variational mean field approximation and describes effective massive gluons propagating in a nonperturbative vacuum. We will use this Fock space here and combine it with nonrelativistic quark an antiquark to compute spectrum of heavy hybrids. 
In [29] we followed the same approach to compute spectrum of gluelumps. By using a single framework offered by the non-relativistic QCD, we will be able to explore the connection between gluelump and hybrid spectra. In this paper we consider the leading contribution to the heavy quarkonium hybrid energies i.e. excluding spin dependent-effects. In the following Section we discuss derivation and treatment of the Foldy-Wouthousen Coulomb gauge (FWCG) Hamiltonian and the mean field approximation to the QCD vacuum. In Section III we summarize calculation of spectrum of ordinary (nonhybrid) quarkonia which is used later to benchmark the hybrid spectrum which is studied in Section IV. Summary and outlook are given in Section V.

\section{FOLDY-WOUTHOUSEN COULOMB GAUGE HAMILTONIAN}

The non-relativistic QCD Hamiltonian, $H_{F W C G}$ describing interaction between heavy quarks and gluons can be constructed from the full QCD Hamiltonian in the Coulomb gauge by employing Foldy-Wouthuysen transformation [18]

$$
H \rightarrow H_{F W C G}=T H T^{-1} .
$$

Here $T=\cdots e^{i S_{2}} e^{i S_{1}} e^{i S_{0}}$ and the hermitian generators $S_{i}$ are of the order $(\langle p\rangle / m)^{i}$ respectively, with $m$ standing for mass of the heavy quarks and $\langle p\rangle$ being the average three-momentum of the heavy quarks. The FWCG Hamiltonian is thus an effective Hamiltonian valid for momenta smaller than the heavy quark mass. The operators $S_{i}$ eliminate couplings between upper and lower components of Dirac spinors to any given order in the $1 / m$ expansion and lead to a Hamiltonian that conserves number of (heavy) quarks. It has the form of a series expansion in inverse powers of the heavy quark mass,

$$
H_{F W C G}=H_{-1}+H_{0}+H_{1}+H_{2}+\cdots
$$

Each term, $H_{i}$ being of order $m^{i}$ depends on the transverse gluon and the non-relativistic quark and antiquark fields. The transverse gluon degrees of freedom are given by the vector potential $\mathbf{A}^{a}(\mathbf{x})$ and the conjugated momentum $\boldsymbol{\Pi}^{a}(\mathbf{x})\left(\boldsymbol{\nabla} \cdot \mathbf{A}^{a}=\boldsymbol{\nabla} \cdot \boldsymbol{\Pi}^{a}=0\right)$ satisfying

$$
\left[\mathbf{A}^{a}(x), \mathbf{\Pi}^{b}(\mathbf{y})\right]=\boldsymbol{\delta}_{T}(\mathbf{x}-\mathbf{y}) \delta^{a b}
$$

where $\left.\boldsymbol{\delta}_{T}(\mathbf{x}-\mathbf{y}) \equiv\left[\mathbf{I}-\boldsymbol{\nabla} \boldsymbol{\nabla} / \boldsymbol{\nabla}^{2}\right] \delta^{3}(\mathbf{x}-\mathbf{y})\right)$. The canonical momentum $\Pi$ is the negative of the transverse component of the chromo-electric field. The quark and antiquark degrees of freedom can be written directly in terms of quark creation and annihilation $\left.Q^{\dagger}, Q\right)$ and antiquark creation and annihilation, $\left(\bar{Q}, \bar{Q}^{\dagger}\right.$ operators which in terms of the original Dirac fields are given by,

$$
\begin{aligned}
& Q_{\lambda}(\mathbf{x}) \equiv\left[\frac{1+\beta}{2} \psi(\mathbf{x})\right]_{\lambda}, \\
& {\left[\bar{Q}(\mathbf{x})\left(-i \sigma_{2}\right)\right]_{\lambda} \equiv\left[\psi^{\dagger}(\mathbf{x}) \frac{1-\beta}{2}\right]_{\lambda+2} .}
\end{aligned}
$$

Here $\lambda=1,2$ denotes the $z$-component of quark spin and the Pauli matrix $\sigma_{2}$ is introduced so that $Q_{\lambda}$ and $\bar{Q}_{\lambda}$ belong the the same $S U(2)$ representation. The mass term $H_{-1}$ is given by (summation over spin indices is implicit)

$$
H_{-1}=m \int d \mathbf{x}\left[Q^{\dagger}(\mathbf{x}) Q(\mathbf{x})+\bar{Q}^{\dagger}(\mathbf{x}) \bar{Q}(\mathbf{x})\right]
$$

The $H_{0}$ term represents the Yang-Mills Hamiltonian coupled to static quark and antiquark sources,

$$
\begin{aligned}
H_{0} & =\frac{1}{2} \int d \mathbf{x}\left[\mathcal{J}^{-1} \mathbf{\Pi}^{a}(\mathbf{x}) \mathcal{J} \mathbf{\Pi}^{a}(\mathbf{x})+\mathbf{B}^{a}(\mathbf{x}) \cdot \mathbf{B}^{a}(\mathbf{x})\right] \\
& +\frac{1}{2} \int d \mathbf{x} d \mathbf{y} \mathcal{J}^{-1} \rho^{a}(\mathbf{x}) K(\mathbf{x}, a ; \mathbf{y}, b) \mathcal{J} \rho^{b}(\mathbf{y})
\end{aligned}
$$

Here $\mathcal{J}=\operatorname{Det}[-\nabla \cdot \mathcal{D}]$ is the determinant of the Faddeev-Popov operator; $\mathcal{D}=\mathcal{D}_{a b}=\delta_{a b} \boldsymbol{\nabla}+g f_{a c b} \mathbf{A}^{c}$ is the covariant derivative in the adjoint representation, and $\mathbf{B}$ is the chromo-magnetic field, $\mathbf{B}^{a}(\mathbf{x})=\boldsymbol{\nabla} \times \mathbf{A}^{a}(\mathbf{x})+$ $(g / 2) f_{a b c} \mathbf{A}^{b}(\mathbf{x}) \times \mathbf{A}^{c}(\mathbf{x})$. The last term in Eq. (6) represent the non-abelian Coulomb gauge interaction between color charge densities, $\rho^{a}(\mathbf{x})=Q^{\dagger}(\mathbf{x}) T^{a} Q(\mathbf{x})-$ $\bar{Q}^{\dagger}(\mathbf{x}) T^{* a} \bar{Q}(\mathbf{x})+f_{a b c} \mathbf{A}^{\mathbf{b}}(\mathbf{x}) \mathbf{\Pi}^{\mathbf{c}}(\mathbf{x})$, and the Coulomb kernel is given by

$$
K(\mathbf{x}, a ; \mathbf{y}, b)=\left[\frac{g}{\nabla \cdot \mathcal{D}}\left(-\nabla^{2}\right) \frac{g}{\nabla \cdot \mathcal{D}}\right]_{(\mathbf{x}, a ; \mathbf{y}, b)} .
$$

The $O(1 / m)$ terms are given by

$$
H_{1}=\frac{1}{2 m} \int d \mathbf{x}\left[Q^{\dagger}(\mathbf{x}) \mathbf{D}^{2}(\mathbf{x}) Q(\mathbf{x})+\left(Q \rightarrow \bar{Q}, T \rightarrow T^{*}\right)\right]-\frac{1}{2 m} \int d \mathbf{x}\left[Q^{\dagger}(\mathbf{x}) g \boldsymbol{\sigma} \cdot \mathbf{B}^{a}(\mathbf{x}) T^{a} Q(\mathbf{x})-\left(Q \rightarrow \bar{Q}, T \rightarrow T^{*}\right)\right]
$$

with $\mathbf{D}$ being the covariant derivative in the fundamental representation, $\mathbf{D}=\mathbf{D}_{i j}(\mathbf{x})=-i \nabla \delta_{i j}-g T_{i j}^{a} \mathbf{A}^{a}(\mathbf{x})$.
The $O(1 / m)$ Hamiltonian arises from the $Z$-graph (see Fig. 1) and is generated by the first order FW transfor- 


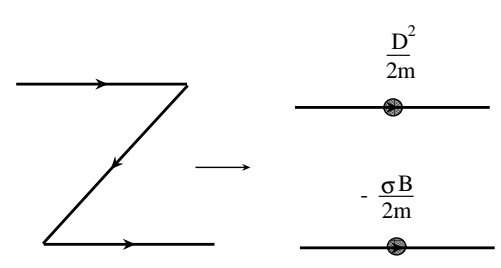

FIG. 1: Non-relativisitc reduction of heavy quark propagator (left side) leads to a kinetic energy term and hyperfine interaction between quark (chromo)magnetic moment and the (chromo)magnetic field.

mation with $S_{1}=(-i / 2 m) \int d \mathbf{x} \psi^{\dagger}(\mathbf{x}) \beta \boldsymbol{\alpha} \cdot \mathbf{D} \psi(\mathbf{x})$. The FW transformation effectively eliminates the quark propagator in Fig. 1 and shrinks the relative distance between the gluon interaction points to zero. This requires that the momentum of the quark propagator is smaller then $m$ thus, as already mentioned, it is necessary that that quark momenta are smaller then the quark mass (this is expected to be the case for low mass heavy quarkonium bound states ). The first term in $H_{1}$ contains quark and anti-quark kinetic energies and $O(1 / \mathrm{m})$ local, spinindependent interaction between (anti)quark and gluon. The second terms describes $O(1 / m)$ spin-dependent local interaction between (anti)quark and gluon. In the leading order we drop the $O(1 / m)$ and higher interactions terms and keep only kinetic energies of the quarks. In Section IV we comment on the results of next to leading order calculation, were we add the remaining $O(1 / m)$ terms. Finally the second term contains $O(1 / m)$ interaction that mixes pure $Q \bar{Q}$ states with $Q \bar{Q} g$, hybrid components. These generate $O\left(1 / \mathrm{m}^{2}\right)$ spin-depdnent splitting which we ignore all together. The final form of $H_{1}$ which is diagonal in the hybrid basis, and is leading order in $O(1 / m)$ is thus given by

$$
\begin{aligned}
H_{1} & =-\frac{1}{2 m} \int d \mathbf{x}\left[Q^{\dagger}(\mathbf{x}) \nabla^{2} Q(\mathbf{x})+\bar{Q}^{\dagger}(\mathbf{x}) \nabla^{2} \bar{Q}(\mathbf{x})\right]+\frac{g^{2}}{2 m} \int d \mathbf{x}\left[Q^{\dagger}(\mathbf{x}) T^{a} T^{b} \mathbf{A}^{a}(\mathbf{x}) \cdot \mathbf{A}^{b}(\mathbf{x}) Q(\mathbf{x})+\left(Q \rightarrow \bar{Q}, T \rightarrow T^{*}\right)\right] \\
& -\frac{g^{2}}{4 m} \int d \mathbf{x}\left[Q^{\dagger}(\mathbf{x}) T^{a} f_{a b c} \boldsymbol{\sigma} \cdot \mathbf{A}^{b}(\mathbf{x}) \times \mathbf{A}^{c}(\mathbf{x}) Q(\mathbf{x})-\left(Q \rightarrow \bar{Q}, T \rightarrow T^{*}\right)\right]
\end{aligned}
$$

with only the first term used in what we define as the leading order calculation.

\section{A. Gluonic degrees of freedom in the mean field}

In the Shrödinger representation the lowest eigenvalue of the QCD Hamiltonian $H_{Q C D}[A] \Psi_{n}[A]=E_{n} \Psi[A]$, $\Psi_{0}[A]$ represents transverse gluon field distribution in the vacuum. In the Coulomb gauge Fock space construction of the excited states (mesons, baryons, hybrids, etc ...) is motivated by the Gribov-Zwaniziger confinement scenario. The vacuum state is expected to be dominated by field configurations near boundary of the Gribov horizon, and gluon excitations which build the hadron spectrum correspond to massive quasi-particles of the harmonic expansion of the the wave functional near the Gribov horizon. In practice, in a mean field description [22-28] the dominance of the field configurations near the Gribov horizon is obtained by choosing the renormalized coupling $g(\Lambda)$ to be such that the inverse of the FaddeevPopov develops a pole near the zero mode. Consider a gaussian ansatz for the vacuum wave functional,

$$
\Psi_{0}[A]=\langle\mathbf{A} \mid 0\rangle \equiv \exp \left(-\frac{1}{2} \int \frac{d \mathbf{k}}{(2 \pi)^{3}} \mathbf{A}^{a}(\mathbf{k}) \omega(k) \mathbf{A}^{a}(\mathbf{k})\right),
$$

in the rainbow ladder approximation, show in Fig. 2 the

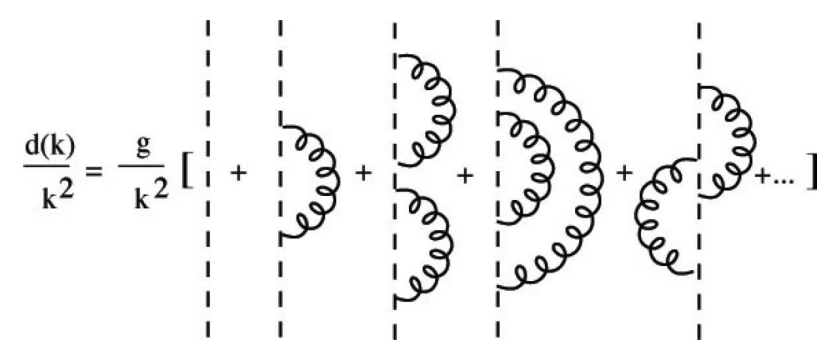

FIG. 2: Diagrammatic representation of the expansion of the functional integral for the ghost propagator c.f. Eq. (10)

expectation value of the inverse Faddeev-Popov operator

$$
\int d \mathbf{x} e^{i \mathbf{k} \cdot \mathbf{x}}\left\langle\Psi_{0}\left|\frac{g}{-\nabla \cdot \mathbf{D}}\right| \Psi_{0}\right\rangle \equiv \frac{d(k)}{k^{2}},
$$

satisfies, $(\hat{\mathbf{k}} \equiv \mathbf{k} / k)$,

$$
\frac{1}{d(k)}=\frac{1}{g(\Lambda)}-\frac{N_{C}}{2} \int^{\Lambda} \frac{d \mathbf{p}}{(2 \pi)^{3}}(1-\hat{\mathbf{k}} \cdot \hat{\mathbf{p}}) \frac{d(|\mathbf{p}-\mathbf{k}|)}{\omega(p)(\mathbf{k}-\mathbf{p})^{2}}
$$

The numerical solution to this Dyson equation has been extensively studied in [22-28]. The main features of 
its solutions remain unchanged if one uses the angular approximation in evaluating the momentum integral $|\mathbf{p}-\mathbf{k}| \rightarrow p \theta(p-k)+k \theta(k-p)$ and use the approximation $\omega(k) \rightarrow m_{g}$ (= constant) for $k \rightarrow 0$ and $\omega(k) \rightarrow k$ for $k>m_{g}[22]$. This approximation enables to obtain an analytical solution to Eq. (11) which is given by

$$
d(k)=\left\{\begin{array}{l}
\frac{g(\Lambda)}{\left[1+\frac{\beta_{L}}{(4 \pi)^{2}} g^{2}(\Lambda)\left(\frac{k}{m_{g}}-\frac{\Lambda}{m_{g}}\right)\right]^{1 / 2}}, k<m_{g} \\
\frac{g(\Lambda)}{\left[1+\frac{\beta_{H}}{(4 \pi)^{2}} g^{2}(\Lambda) \log \left(\frac{k^{2}}{m_{g}^{2}}\right)\right]^{1 / 2}}, k>m_{g}
\end{array}\right.
$$

with $\beta_{H}=8 N_{C} / 3$ and $\beta_{L}=5 \beta_{L} / 3$. It follows that $d(k)$ can be made renormalization scale invariant by choosing $g(\Lambda)$ to depend on $\Lambda$ in the same way $d(k)$ depends on $k$. This also allows for interpreting $d(k)$ as the running coupling. For $g(\Lambda) \leq g_{C} \equiv 4 \pi\left(m_{g} / \beta_{L} \Lambda\right)^{1 / 2}$ the Landau pole is avoided and, as $g(\Lambda) \rightarrow g_{C}$ from below, the functional integral of the gluon field in Eq. (10) becomes dominated by the configurations near the Gribov horizon. The parameter $\omega$ which fixes the vacuum wave functional can be constrained by minimizing the energy density $\delta\left\langle\Psi_{0}|H| \Psi_{0}\right\rangle=0$.
The resulting gap equation involves expectation value of the Faddeev-Popov operator whose computation, however is not free of from ambiguities. These ambiguities arise of UV subtraction which can be absorbed into definition of $\omega[25,28]$. Therefore we choose a more phenomenological approach. Following our studies of the gluelump spectrum [29] we will treat $\omega$ as a parameter of the model to be constrained by the physical spectrum rather then by the properties of the vacuum i.e. the gap equation. Since the gluon 2-point function is given in terms of $\omega$, by

$$
\left\langle\Psi_{0} \mid \mathbf{A}^{a}(\mathbf{k}) \mathbf{A}^{b}(\mathbf{p})\right\rangle=(2 \pi)^{3} \delta(\mathbf{k}+\mathbf{p}) \frac{\delta_{a b}}{2 \omega(k)}
$$

it is clear that the wave functional in Eq. (9) leads to a gap in single gluon spectrum. The energy of the single gluon state defined as

$$
|1 g\rangle=|\mathbf{k}, \lambda, a\rangle=a^{\dagger}(\mathbf{k}, \lambda, a)\left|\Psi_{0}\right\rangle
$$

where the single gluon creation (annihilation) operators $a^{\dagger},(a)$ are defined by ( $\boldsymbol{\epsilon}$ are helicity vectors)

$$
\begin{gathered}
\mathbf{A}^{a}(\mathbf{x})=\int \frac{d \mathbf{k}}{(2 \pi)^{3}} \frac{1}{\sqrt{2 \omega(k)}}\left[\boldsymbol{\epsilon}(\mathbf{k}, \lambda) a(\mathbf{k}, \lambda, a)+\boldsymbol{\epsilon}^{\dagger}(-\mathbf{k}, \lambda) a^{\dagger}(-\mathbf{k}, \lambda)\right] e^{i \mathbf{k} \cdot \mathbf{x}} \\
\boldsymbol{\Pi}^{a}(\mathbf{x})=-i \int \frac{d \mathbf{k}}{(2 \pi)^{3}} \sqrt{\frac{\omega(k)}{2}}\left[\boldsymbol{\epsilon}(\mathbf{k}, \lambda) a(\mathbf{k}, \lambda, a)-\boldsymbol{\epsilon}^{\dagger}(-\mathbf{k}, \lambda) a^{\dagger}(-\mathbf{k}, \lambda)\right] e^{i \mathbf{k} \cdot \mathbf{x}}
\end{gathered}
$$

is given by $[22,23]$

$$
\frac{\Sigma_{g}(k)}{\omega(k)}=1-\frac{N_{c}}{2}(2 \pi) \int \frac{d \mathbf{k}^{\prime}}{(2 \pi)^{3}} \widetilde{V}_{C L}\left(\left|\mathbf{k}-\mathbf{k}^{\prime}\right|\right) \frac{1+\left(\hat{\mathbf{k}} \cdot \hat{\mathbf{k}}^{\prime}\right)^{2}}{2 \omega\left(k^{\prime}\right)} .
$$

Here $\widetilde{V}_{C L}$ is the negative of the momentum space expectation value of the Coulomb potential

$$
\widetilde{V}_{C L}(k)=\int d \mathbf{x} e^{i \mathbf{k} \cdot \mathbf{x}}\left\langle\Psi_{0}\left|\frac{g}{\nabla \cdot \mathbf{D}}\left(\nabla^{2}\right) \frac{g}{\nabla \cdot \mathbf{D}}\right| \Psi_{0}\right\rangle
$$

which is well approximated by a combination of Coulomb and linear potential [22]. The long range part of $V_{C L}$ can potentially lead to and IR divergence in the self energy ( integral in Eq. (16)) from the integration region $\mathbf{k}^{\prime} \sim \mathbf{k}$. This divergence is however canceled in the full bound state equation by similar divergencies arising from quark self energies and quark-antiquark interaction. The quadratic UV divergence from $\mathbf{k}^{\prime} \rightarrow \infty$ in the self energy integrals is canceled by a gluon mass counterterm $[22,23]$. In the basis of massive gluons the Foldy-
Wouthousen Hamiltonian is schematically given by

$$
H_{F W C G}=\sum_{\mathbf{k}, \lambda, a} \Sigma_{g}(k) a^{\dagger}(\mathbf{k}, \lambda, a) a(\mathbf{k}, \lambda, a)+V\left(a^{\dagger}, a\right)
$$

where $V$ represents residual interactions between gluons and heavy quarks. The gluon mass gap implied by $\Sigma_{g}(k) \neq k$ suggests that a hybrid state in which quarks and gluons have residual 3-momenta of the order of $\Lambda_{Q C D}$ should be well approximated by a state containing a pair of non-relativisitc quark and antiquark and a single quasi-gluon. In the infinite heavy quark mass limit, separation between the quarks becomes a good quantum number and energy spectrum of such static hybrids is determined by the energy of the single quasi-gluon in presence of color octet $Q \bar{Q}$ source. As separation between quarks is taken to zero spectrum of static hybrids becomes rotationally invariant and such states are being referred to as gluelumps. Lattice simulations show that the lowest glue-lump state has spin J, parity $\mathrm{P}$, and charge conjugation $\mathrm{C}, J^{P C}=1^{+-}$and the first excited state, $J^{P C}=1^{--}$. This unusual parity inversion between vec- 
tor and pseudo-vector states has been explained using the Coulomb gauge approach as due to the three-body force involving all three particles quark, antiquark and gluon originating from the non-abelian Coulomb interaction in Eq. (6). A good agreement between our Coulomb gauge approach and lattice gluelump and static hybrid spectra gives us confidence that the approach may also be adequate for computation of the spectrum of hybrids with dynamical heavy quarks.

\section{ORDINARY QUARKONIA}

Before discussing gluonic excitations we shall fix the parameters of the $Q \bar{Q}$ sector by comparing the $H_{F W C G}$ Hamiltonian spectrum with that of ordinary charmonia.

\section{A. Basis and Hamiltonian matrix elements}

The Coulomb gauge Foldy-Wouthuysen Hamiltonian should reproduce the known spectrum of ordinary quarkonia. Since low-lying quarkonia are expected to be dominated by the $Q \bar{Q}$ component we may represent $N$-th quarkonium state of spin and $z$-component $J, M$ parity $P$ and charge conjugation $C$, as

$$
|J M P C N\rangle=\sum_{\alpha \equiv\left(S_{q}, L_{q}\right)} \int \frac{q^{2} d q}{(2 \pi)^{3}} \Psi_{\alpha}^{N}(q)|J M P C ; \alpha ; q\rangle
$$

with the $Q \bar{Q}$ state given by

$$
\begin{aligned}
|J M P C ; \alpha ; q\rangle & =\sum_{m_{1}, m_{2}} \int d \hat{\mathbf{q}} \chi_{m_{1}, m_{2}}^{J M P C}(\hat{\mathbf{q}}, \alpha) \\
& \times Q_{\mathbf{q}, m_{1}, i_{1}}^{\dagger} \frac{\delta_{i_{1}, i_{2}}}{\sqrt{N_{c}}} \bar{Q}_{-\mathbf{q}, m_{2}, i_{2}}^{\dagger}|0\rangle,
\end{aligned}
$$

and where the spin-orbial wave function $\chi$ describes coupling of total quark spin $S_{q}$ to relative orbital angular momentum $L_{q}$ to total spin of the quarkonium $J$, $\mathbf{L}_{q}+\mathbf{S}_{q}=\mathbf{J}$

$$
\chi_{m_{1}, m_{2}}^{J M P C}(\hat{\mathbf{q}}, \alpha)=\frac{1}{2}\left[1+C(-1)^{L_{q}+S_{q}}\right] \frac{1}{2}\left[1+P(-1)^{L_{q}+1}\right] \sum_{M_{S}, M_{L}}\left\langle\frac{1}{2} m_{1} ; \frac{1}{2} m_{2} \mid S_{q} M_{S}\right\rangle\left\langle S_{q} M_{S} ; L_{q} M_{L} \mid J M\right\rangle Y_{L_{q} M_{L}}(\mathbf{q}) .
$$

The Shrödinger equation for the masses of ordinary quarkonia is given by

$$
\begin{aligned}
M_{N} \Psi_{\alpha}^{N}(q) & =\left[2 m+\frac{q^{2}}{m}+\Sigma_{q}\right] \Psi_{\alpha}^{N}(q) \\
& +C_{F} \sum_{\alpha^{\prime}} \int \frac{q^{\prime 2} d q^{\prime}}{(2 \pi)^{3}} V_{Q \bar{Q}}\left(q, \alpha ; q^{\prime} \alpha^{\prime}\right) \Psi_{\alpha^{\prime}}^{N}\left(q^{\prime}\right)
\end{aligned}
$$

The first term represents quark and antiquark kinetic energies and their self-energies,

$$
\Sigma_{q}=-C_{F} V_{L}(0)=-C_{F} \int \frac{d \mathbf{q}}{(2 \pi)^{3}} \widetilde{V}_{L}(q)
$$

In position space the expectation value of the Coulomb potential given in Eq. (17) leads to a short range part $V_{C L}(R) \rightarrow V_{C}(R) \propto-1 / R$ for $R \rightarrow 0$ and long range part, $V_{C L}(R) \rightarrow V_{L}(R) \propto R$ for $R \rightarrow \infty$ The contribution from the short range part is absorbed in the definition of the quark mass. The long range part leads to an IR divergence which in momentum space and is represented by the constant $V_{L}(0)$ in Eq. (23). This constant, and therefore sensitivity to the $R \rightarrow \infty$ limit of the position space Coulomb interaction is removed by the long-range part of the quark-antiaquark potential,

$$
V_{Q \bar{Q}}=\delta_{\alpha^{\prime} \alpha} \int d^{2} \hat{\mathbf{q}}^{\prime} P_{L}\left(\hat{\mathbf{q}}^{\prime} \cdot \hat{\mathbf{q}}\right) \widetilde{V}_{C L}\left(\left|\mathbf{q}^{\prime}-\mathbf{q}\right|\right)
$$

as it should for color singlet bound states.

We use the quarkonium spectrum to determine the renormalized quark mass. From experimental data we find for the the $S$-wave $\left(L_{q}=0\right)$ spin-averaged masses

$$
\bar{M}^{S}=\frac{1}{4}\left[M_{0^{-+}}+3 M_{1^{--}}\right]
$$

$\bar{M}_{c \bar{c}}^{S}=3.068 \mathrm{Gev}$ for charmonium and $\bar{M}_{b \bar{b}}^{S}=9.46$ Gev for bottomonium, respectively. Comparing with results of numerical solution to Eq. (22) we extract $m_{c}=$ $1.16 \mathrm{GeV}$ and $m_{b}=4.58 \mathrm{GeV}$, respectively. In Fig. 3 we compare the predicted masses of the first radial excitation of the $S$-waves and the ground state (spin-averaged) $P$-waves with experimental data. The observed agreement is a reflection of the Coulomb gauge kernel correctly reproducing the "Coulomb + Linear" potential in the region of position space covered by the bound quarkonium wave functions.

\section{SPECTRUM OF GLUONIC EXCITATIONS}

As discussed above, gluelumps provide a good benchmark for the spectrum of gluonic excitations. Upon neglecting the quark motion (i.e setting $H_{1}=0$ ) (anti)quark positions become good quantum numbers, 
and in particular the spectrum of $Q \bar{Q}$ states with vanishing relative separation between quark sources is given by the energy of gluon cloud. In our approach the low lying spectrum is obtained by binding a single quasi gluon with kinetic energy given by Eq. (16) to the $Q \bar{Q}$ source through the Coulomb kernel given in Eq. (6). More details are given in Ref. [20, 21, 29]. The spin of the gluelump is then identified with the total angular momentum $J_{g}$ of the quasi-gluon, orbiting the static $Q \bar{Q}$ source. Once the sources are allowed to move the total spin of the resulting hybrid meson is given by the sum of angular momenta of all constituents. Since heavy quarks are expected to move non-relativisitcaly with average separation of the order of $O\left(1 / \alpha_{s} m\right)$ it is reasonable to expect there will be not much distortion of the gluon wave function of the gluelump due to quark motion. Finely, since we are ignoring quark-spin dependent interactions it will be useful to couple quark spin at the very last to other angular momenta before producing a state of good total $\operatorname{spin} J$.

\section{A. Basis and Hamiltonian matrix elements}

From the above discussion it follows that the optimal basis of $Q \bar{Q} g$ states is such in which the $Q \bar{Q}$ relative angular momentum $L_{q}$ is first coupled to total gluon spin $J_{g}$. The resulting angular momentum $j$ is then coupled to the total quark-antiquark spin $S_{q}$ to the give the total spin of the hybrid state $J$. In this coupling scheme it is straightforward to take the static quark limit and thus compare hybrid and gluelump spectra. Specifically, the $N$-th hybrid state with total spin and its $z$-axis projection, $J, M$, parity $P$, charge conjugation $C$ is given by

$$
\begin{aligned}
& |J M P C N\rangle=\sum_{\alpha \equiv\left(\sigma, S_{q}, L_{q}, J_{q}\right)} \int \frac{k^{2} d k}{(2 \pi)^{3}} \frac{q^{2} d q}{(2 \pi)^{3}} \Psi_{\alpha}^{N}(k, q) \\
& \times|J M P C ; \alpha ; k, q\rangle \text {. }
\end{aligned}
$$

Here the quark-antiquark-gluon, $Q \bar{Q} g$ state is given by

$$
\begin{gathered}
|J M P C ; \alpha ; k, q\rangle=\sum_{m_{1}, m_{2}, \sigma} \int d \hat{\mathbf{k}} d \hat{\mathbf{q}} \chi_{m_{1}, m_{2}, \sigma}^{J M P C}(\hat{\mathbf{k}}, \hat{\mathbf{q}}, \alpha) \\
\times Q_{\frac{\mathbf{k}}{2}+\mathbf{q}, m_{1}, i_{1}}^{\dagger} \frac{T_{i_{1}, i_{2}}^{a}}{\sqrt{C_{F} N_{c}}} \bar{Q}_{\frac{\mathbf{k}}{2}-\mathbf{q}, m_{2}, i_{2}}^{\dagger} a_{-\mathbf{k}, \sigma, a}^{\dagger}|0\rangle .
\end{gathered}
$$

The spin-orbital wave function $\chi$ describes the $\left(\mathbf{L}_{\mathbf{q}}+\mathbf{J}_{q}\right)+$ $\mathbf{S}_{q}$ coupling discussed above, and $\sigma= \pm 1$ represents gluon helicity

$$
\begin{aligned}
& \chi_{m_{1}, m_{2}, \sigma}^{J M P C}(\hat{\mathbf{k}}, \hat{\mathbf{q}}, \alpha)=\sqrt{\frac{2 J_{g}+1}{4 \pi}} \frac{1}{2}\left[1+C(-1)^{L_{q}+S_{q}+1}\right] \\
\times & \frac{1}{\sqrt{2}}(-1)^{J_{g}} \sum_{M_{S}, M_{L}, m}\left\langle\frac{1}{2} m_{1} ; \frac{1}{2} m_{2} \mid S_{q} M_{S}\right\rangle \\
\times & \left\langle J_{g} M_{g} ; L_{q} M_{L} \mid j m\right\rangle\left\langle j m ; S_{q} M_{S} \mid J M\right\rangle Y_{L_{q} M_{L}}(\mathbf{q}) \\
\times & D_{M_{g},-\sigma}^{* J_{g}}(\hat{\mathbf{k}})\left[\delta_{\sigma, 1}+P(-1)^{J_{g}+L_{q}+1} \delta_{\sigma,-1}\right] .
\end{aligned}
$$

Finally, $\mathbf{q}$ is the relative momentum between the quark-antiquark and $\mathbf{k}$ is the momentum of the gluon in the hybrid center of mass frame. The spin-orbtal wave function is kinematical, i.e. determined by the rotational symmetry of $H_{F W C G}$ Hamiltonian, while the radial wave function $\Psi_{\alpha}^{N}(k, q)$ will be determined by diagonalizing the Schrödinger equation for the hybrid bound state. Parity and charge conjugation are also kinematical and given by

$$
\begin{aligned}
& P=\xi(-1)^{J_{g}+L_{q}+1}, \\
& C=(-1)^{L_{q}+S_{q}+1},
\end{aligned}
$$

respectively. Here $\xi=+1$ for TM (natural parity) and $\xi=-1$ for TE ( unnatural parity ) gluon state which correspond to a $|\sigma=+1\rangle+\xi|\sigma=-1\rangle$ combination of gluon helicity states. We note that, as expected, both $P$ and $C$ are a product of $Q \bar{Q}$ and gluelump parity and charge conjugation given by

$$
\begin{aligned}
& P_{q}=(-1)^{L_{q}+1}, P_{g}=\xi(-1)^{J_{g}}, \\
& C_{q}=(-1)^{L_{q}+S_{q}}, C_{g}=-1 .
\end{aligned}
$$

The Schrödinger equation for the radial wave function has the form of 


$$
\begin{aligned}
{\left[2 m+\frac{q^{2}}{m}+\frac{k^{2}}{4 m}+\Sigma_{g}(k)+\Sigma_{q}\right] \Psi_{\alpha}^{N}(k, q) } & -\frac{1}{2 N_{C}} \sum_{\alpha^{\prime}} \int \frac{q^{\prime 2} d q^{\prime}}{(2 \pi)^{3}} V_{Q \bar{Q}}\left(q, \alpha ; q^{\prime}, \alpha^{\prime}\right) \Psi_{\alpha^{\prime}}^{N}\left(k, q^{\prime}\right) \\
& +\sum_{\alpha^{\prime}} \int \frac{k^{\prime 2} d k^{\prime}}{(2 \pi)^{3}} \frac{q^{\prime 2} d q^{\prime}}{(2 \pi)^{3}} V_{Q \bar{Q} g}\left(k, q, \alpha ; k^{\prime}, q^{\prime}, \alpha^{\prime}\right) \Psi_{\alpha^{\prime}}^{N}\left(k^{\prime}, q^{\prime}\right)=M_{N} \Psi_{\alpha}^{N}(k, q) .
\end{aligned}
$$

In leading order the fist term represents kinetic energies and self-energies of the gluon, given by Eq. (16), and of quarks given by Eq. (23). As in the case of Eq. (22) the IR divergencies in the self energies are canceled by the potential matrix elements. The first integral in Eq. (31) is the $Q \bar{Q}$, interaction. It is independent of the gluon degrees of freedom and repulsive in for the $Q \bar{Q}$ pair in the color octet. Finally, the last term on the left hand side of Eq. (31) represents collectively the two body attrac- tive interactions between the quark and the gluon and the antiquark and the gluon and the irreducible threebody interaction linking all three constituents - quark, antiquark and gluon,

$$
V_{Q \bar{Q} g}=V_{Q \bar{Q} g}^{2}+V_{Q \bar{Q} g}^{3}
$$

The two-body interaction is given by

$$
\begin{aligned}
V_{Q \bar{Q} g}^{2}\left(k, q, \alpha ; k^{\prime}, q^{\prime}, \alpha\right) & =\frac{N_{c}}{4}\left(\sqrt{\frac{\omega(k)}{\omega\left(k^{\prime}\right)}}+\sqrt{\frac{\omega\left(k^{\prime}\right)}{\omega(k)}}\right) \sum_{m_{1}, m_{2}, \sigma, \sigma^{\prime}} \int d \hat{\mathbf{k}} d \hat{\mathbf{q}} d \hat{\mathbf{k}}^{\prime} d \hat{\mathbf{q}}^{\prime} \chi_{m_{1}, m_{2}, \sigma}^{* J M P C}(\hat{\mathbf{k}}, \hat{\mathbf{q}}, \alpha) \chi_{m_{1}, m_{2}, \sigma^{\prime}}^{J^{\prime} M^{\prime} P^{\prime} C^{\prime}}\left(\hat{\mathbf{k}}^{\prime}, \hat{\mathbf{q}}^{\prime}, \alpha^{\prime}\right) \\
& \times\left[\delta\left(\mathbf{q}+\mathbf{k} / 2-\mathbf{q}^{\prime}-\mathbf{k}^{\prime} / 2\right)+\delta\left(-\mathbf{q}+\mathbf{k} / 2+\mathbf{q}^{\prime}-\mathbf{k}^{\prime} / 2\right)\right] \widetilde{V}_{C L}\left(\mathbf{k}-\mathbf{k}^{\prime}\right) \epsilon_{-\mathbf{k}^{\prime}, \sigma^{\prime}} \cdot \epsilon_{-\mathbf{k}, \sigma}^{*}
\end{aligned}
$$

The three body interaction emerges from the non-abelian Coulomb kernel in Eq. (7). The general expression for the three body interaction, $V_{Q \bar{Q} g}^{3}$ is quite complicated, and we discuss special cases in the Appendix. The matrix elements of next to leading order interactions are also given in the Appendix.

\section{B. Hybrid vs gluelump spectra}

The particular choice of the coupling of spin and orbital degrees of freedom in Eq. (28) enables to make a direct contact with the gluelump spectrum. In that case the orbital part of the $Q \bar{Q}$ wave function is in the $S$-wave and the radial wave function is proportional to a $\delta$-function in coordinate space. We take the radial part as a normalized gaussian,

$$
\Psi_{Q}^{S}(q)=\frac{2}{\pi^{\frac{1}{4}}}\left(\frac{2 \pi}{\alpha_{Q}}\right)^{\frac{3}{2}} e^{-\frac{q^{2}}{2 \alpha_{Q}^{2}}}, \quad \int \frac{q^{2} d q}{(2 \pi)^{3}}\left|\Psi_{Q}^{S}(q)\right|^{2}=1 .
$$

The leading order Hamiltonian truncated to the $S$-wave quark-antiquark orbital is given in the Appendix. It is straightforward to verify that removing the quark mass term and taking the limit $\alpha_{Q} \rightarrow \infty($ after $m \rightarrow \infty)$ in Eq. (31), which corresponds to setting the relative quark separation to zero, the gluelump Schrödindger equation from [29] is reproduced.

With dynamical quarks the quark-gluon interaction mixes the quark orbital angular momentum with gluon spin while in leading order it preserves quark spin. This is represented by a mixing between $\alpha$ and $\alpha^{\prime}$ indices. To leading order, however, quark spin is conserved, $S_{q}=S_{q}^{\prime}$ and then parity and charge conservation imply no mixing between TE and TM gluons. As long as the quark motion does not distort much the gluon distribution of a gluelump one would expect mixing between various $\left(L_{q}, J_{g}\right)$ states to be small. Spin splitting is also expected to be small, of the order of $O(1 / m)$. In other words spectrum of low hybrids are expected to be approximately classified by the product of gluelump and $Q \bar{Q}$ quantum numbers. In the following we will compute the hybrid spectrum under this approximation. Do not see much point in going beyond this approximation. This because we expect uncertainty in the spectrum associated with our mean field treatment of gluonic degrees of freedom to be of similar order of magnitude. This will be shown in Section IV C were we study dependence of the hybrid spectrum on the mean field parameter, $\omega$ ( $c f$. Eq. (9)). We also restrict our study to lowest multiplets, since bare bound state spectrum is expected to be renormalized above open decay channels.

The two lowest $\left(L_{q}, J_{g}\right)$ multiples , $\left(L_{q}, J_{q}\right)=$ $(0,1),(1,1)$ lead to the following set of states. For $J_{g}=1$ 
there two possible gluelumps, the ground state with $J_{g}^{P C}=1^{+-}$and gluon in the TE mode and the first excited gluelump state with $J_{g}^{P C}=1^{--}$and the TM gluon. Coupling the TE gluon with the color octet $Q \bar{Q}$ state in $L_{q}=0, S$-wave orbital leads to a hybrid state with the intermediate angular momentum $\mathbf{j}=\mathbf{L}_{q}+\mathbf{J}_{q}=1$. After adding the quark spin $S_{q}=0,1$, in absence of spin-dependent interaction and weak mixing between different quark orbitals, we obtain four low lying hybrids with quantum numbers, $J^{P C}=1^{--}$for $S_{q}=0$ and $J^{P C}=0^{-+}, 1^{-+}, 2^{-+}$for $S_{q}=1$. It is worth noting that hybrid with exotic quantum numbers $1^{-+}$appears in this lowest multiplet and is predicted to have the $Q \bar{Q}$ pair in spin-1. The TM gluon coupled to the octet $Q \bar{Q}$ state would lead to four hybrids with $J^{P C}=1^{+-}$for $S_{q}=0$ and $J^{P C}=0^{++}, 1^{++}, 2^{++}$for $S_{q}=1$. The $L_{q}=1 Q \bar{Q}$ multiplet, is expected to be at a similar energy if orbital excitations of the quarks is energetically comparable to orbital excitation of the quasi-gluon.

\section{Numerical Results}

Solving the radial Shrödinger equation, (31), even for fixed $L_{q}, J_{g}$ is quite challenging numerically. We proceed with the following numerical approximation. First we use a single harmonic oscillator quark wave function to solve for the low-lying gluon energy in the background of either $S\left(L_{q}=0\right)$ or $P\left(L_{q}=1\right)$ wave quarks. The size of the quark wave function is determined by minimizing the total hybrid energy. In the next step and to validate single harmonic oscillator approximation for the quark wave function, we use the solution for the gluon wave function from the first step and solve for the quark wave function. We find variation in the hybrid energies to be less then a percent. Taking advantage of the gaussian quark wave function, we can introduce an effective potential for the gluon field and, as discussed earlier make the connection between hybrid and gluelump states. In this approximation hybrid can be viewed as a single quasi-gluon moving in an effective potential originating from the slow moving quarks. In the Appendix we give explicit formulas for the gluon interactions involving $S$-wave quarks.

In Fig. 3 full boxes show our results for the spinaveraged masses of ordinary quarkonia. As discussed in Sec. III the $0^{-+}$and $1^{--}$states were used to fix the quark quark mass. We find the first radial $S$-wave excitation at $M_{0^{\prime-+}}=3.82 \mathrm{GeV}$ and the center of mass of the $P_{-}$ waves at $M_{1^{+-}}=M_{0^{++}}=M_{1_{++}}=M_{2^{++}}=3.53 \mathrm{GeV}$, which compare favorably to the experimental values as seen from Table I.

As discussed in [29] we determine gluon mean field parameter $\omega(k)$ which appears in the ansatz for the vacuum, Eq. (9) by fitting the gluelump spectrum. Using $\omega(k)$ specified by model-3 in [29] for the masses of lowest two gluelump states $J_{g}^{P C}=1^{+-}$and $1^{--}$, gives $0.89 \mathrm{GeV}$ and $1.29 \mathrm{GeV}$ compared to lattice results of 0.87(15) Gev and 1.25(16) Gev, respectively. In compar-

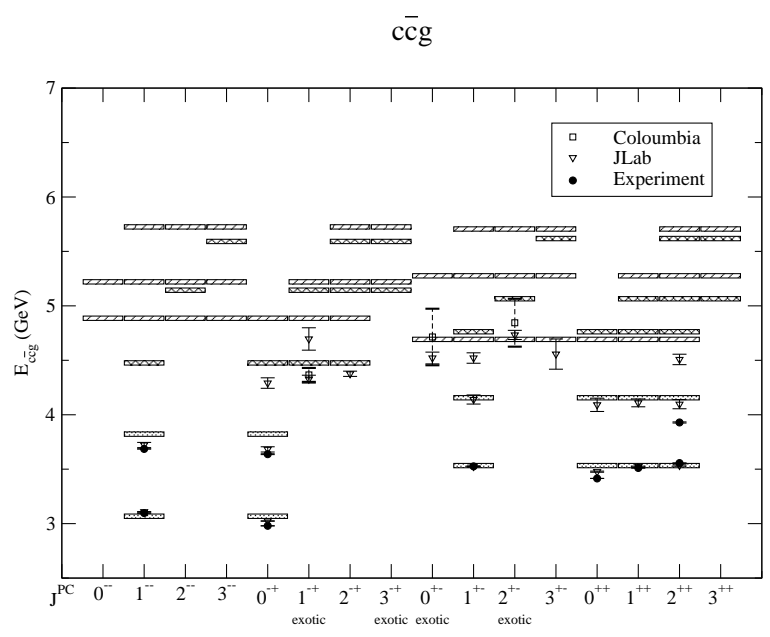

FIG. 3: Charmonium (solid boxes) and charmonium hybrid spectrum compared with data (where available) or lattice computations. Single dashed boxes are the $c \bar{c} g$ hybrids dominated by the $P$-wave quarks, all other have the $Q \bar{Q}$ pair in the relative $S$-wave orbital.

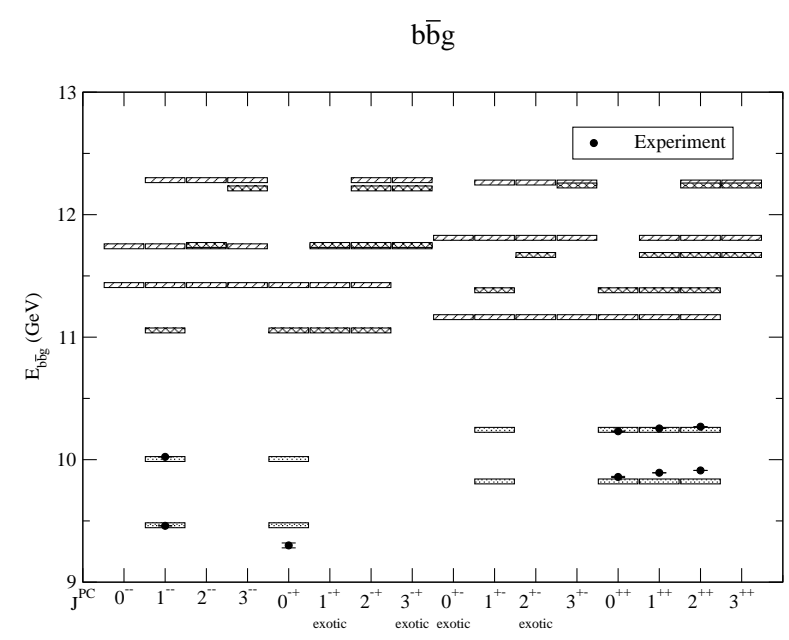

FIG. 4: Same as Fig. 3 for bottomonium.

ison, the original vacuum model of Ref. [22, 23], referred to in [29] as model-1 results in higher gluelump masses of $2.05 \mathrm{GeV}$ and $2.19 \mathrm{GeV}$, respectively.

We can use the gluelump and ordinary $Q \bar{Q}$ masses to obtain a simple estimate for the hybrid spectrum. For hybrids with the TE gluon i.e. built on top of the ground state, $1^{+-}$gluelump and the $S$-wave $Q \bar{Q}$ state we expect the exotic $1^{-+}$charmonium hybrid at $M_{1^{-+}} \sim m_{g}+$ $2 m_{Q}+m_{Q}\left\langle v^{2}\right\rangle \sim 0.87(2.05)+2.3+0.5=3.67(4.85) \mathrm{GeV}$ for model-3 (model-1) were we used $\left\langle v^{2}\right\rangle \sim 0.4 \sim 0.5$. 
TABLE I: $Q \bar{Q}$ charmonium spectrum

\begin{tabular}{|l|c|c|c|c|c|}
\hline$J^{P C}$ & Exp. $[\mathrm{GeV}]$ & This work $[\mathrm{GeV}]$ & $J^{P C}$ & Exp $[\mathrm{GeV}]$ & This work $[\mathrm{GeV}]$ \\
\hline $0^{-+}$ & $2.980(1)$ & 3.07 & $0^{\prime-+}$ & $3.637(4)$ & 3.82 \\
\hline $1^{--}$ & $3.097(0)$ & 3.07 & $1^{\prime--}$ & $3.686(0)$ & 3.82 \\
\hline $1^{+-}$ & $3.526(0)$ & 3.53 & $1^{\prime+-}$ & - & 4.16 \\
\hline $0^{++}$ & $3.415(0)$ & 3.53 & $0^{\prime++}$ & - & 4.16 \\
\hline $1^{++}$ & $3.511(0)$ & 3.53 & $1^{\prime++}$ & - & 4.16 \\
\hline $2^{++}$ & $3.556(0)$ & 3.53 & $2^{\prime++}$ & $3.929(5)$ & 4.16 \\
\hline
\end{tabular}

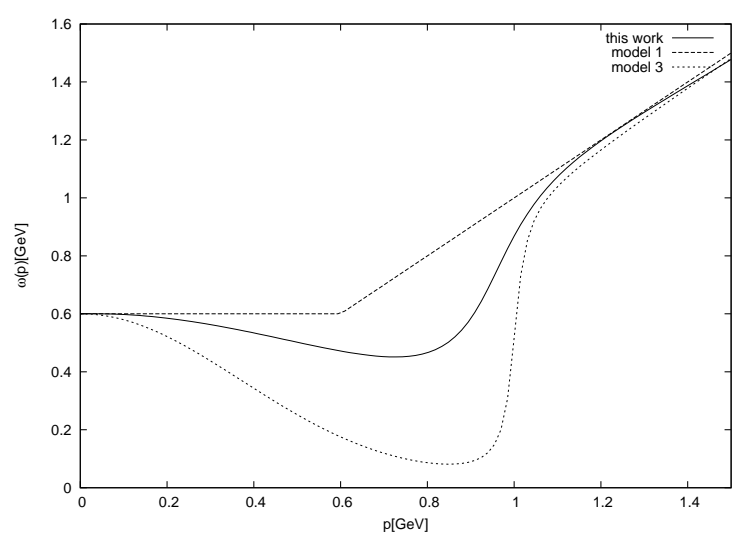

FIG. 5: Vacuum wave functional parameter $\omega(k)$, model-1 and model-3 were discussed in [29]. The dotted line is used here and give the best fit to the lowest gluelump and $1^{-+}$ hybrid.

The recent lattice calculation of [14] palce the $1^{-+}$ charmonium exotic at $4.33 \mathrm{GeV}$, i.e. between our two models of the vacuum wave functional. Thus finally we vary $\omega(k)$ to obtain masses closest to both the ground state gluelump and the $1^{-+}$hybrid. The corresponding $\omega$ is shown in Fig. 5 for which we find the $1^{-+}$ hybrid at $4.47 \mathrm{GeV}$ and the two lowest gluelumps at $M_{1^{+-}}=1.72 \mathrm{Gev}$ and $M_{1^{--}}=2.12 \mathrm{Gev}$.

The final results for the charmonium and bottomonium spectrum are shown in Fig. 3 and Fig. 4 respectively. As discussed earlier, if mixing between $\left(L_{q}, J_{g}\right)$ multiplets is excluded and with hyperfine interactions turned off we find $4 Q \bar{Q} g$ degenerate states for $L_{g}=0$ and 12 degenerate states for $L_{g}=1$. We have computed the effects of $O(1 / m)$ interactions which include spin dependent terms and find them to contribute at the level of $30-50 \mathrm{MeV}$ to the charmonium states which is certainly below the accuracy of the variations in the mean field wave functional. Our results are compared to experimental data and/or lattice calculations where available. The particular degeneracy pattern we find seems to suggest that the $P C=-+$ triplet of states found in lattice simulations at $4.3 \mathrm{GeV}$ does indeed contain the $1^{-+}$exotic [30]. In Tables II,III we give our predictions for the masses of the $c \bar{c}$ g hybrids and the possible identification with the

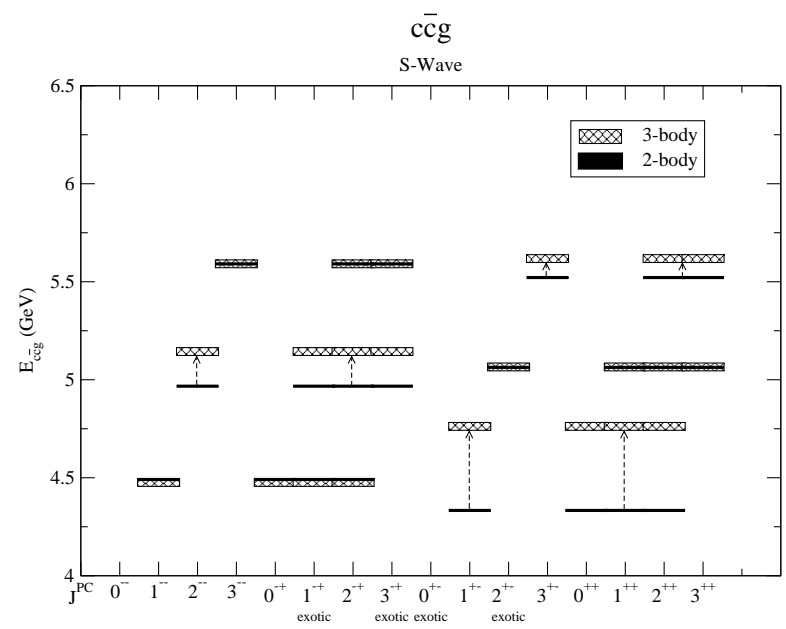

FIG. 6: The effect of the irreducible three body potential on the charmonium hybrid spectrum with $S$-wave quarks. Sold lines represent the spectrum form Eq. (31) with $V^{3}$ in Eq. (32) removed.

lattice states from [14].

Finally in Fig. 6 we show the effect of the irreducible three body interaction, $V_{Q Q g}^{3}$, that is germane to the Coulomb gauge. It is this interaction that is responsible for producing the inverted parity ordering of the gluelump spectra, i.e producing the $1^{+-}$gluelump below the $1^{--}$. It leads to analogous parity-inversion in the $Q \bar{Q} g$ quarkonium spectra, i.e. it leads to the $1^{--}, 0^{-+}, 1^{-+}, 2^{-+}$multiplet below $1^{+-}, 0^{++}, 1^{++}, 2^{++}$.

\section{SUMMARY AND OUTLOOK}

We studied spectrum of heavy quarkonium hybrids in Coulomb gauge QCD with gluon degrees of freedom in the mean field approximation. In general, we have found a reasonable agreement with lattice data. In particular we find the $1^{-+}$exotic charmonium state at $4.47 \mathrm{GeV}$. Our predictions seem to be systematically higher compared to lattice which is most likely a reflection of a deficiency of the gaussian, mean filed, vacuum wave func- 
TABLE II: Charmonium hybrids with $S$-wave $Q \bar{Q}$ pair compared with lattice results were avilable. The second column shows masses all states in a given multiplet (third column) from the Coulomb gauge calculation. In the forth column we give the lattice results that are closest to our predictions. The states marked by [?] (if exist) have not been resolved on the lattice. Lattice computations find lower mass states for these quantum numbers. it is possible that these could be interpreted as $Q \bar{Q}$ states. The exception is the $3^{-+}$for which no lattice state was found.

\begin{tabular}{|c|c|c|c|}
\hline$J_{g}^{P_{g}}$ & This work $[\mathrm{GeV}]$ & $J^{P C}$ & Lattice $[14][\mathrm{GeV}]$ \\
\hline $1^{+}$ & 4.476 & $0^{-+}, 1^{-+}, 2^{-+},\left[1^{--}\right]$ & $4.291(48), 4.327(36), 4.376(24),[?]$ \\
\hline $1^{-}$ & 4.762 & $1^{+-}, 2^{++},\left[0^{++}, 1^{++}\right]$ & $4.521(48), 4.508(48),[?, ?]$ \\
\hline $2^{+}$ & 5.144 & $1^{-+},\left[2^{--}, 2^{-+}, 3^{-+}\right]$ & $4.696(103),[?, ?, ?]$ \\
\hline $2^{-}$ & 5.065 & $2^{+-},\left[1^{++}, 2^{++}, 3^{++}\right]$ & $4.733(42),[?, ?, ?]$ \\
\hline
\end{tabular}

TABLE III: Same as in Table II for $Q \bar{Q}$ in the $P$-wave

\begin{tabular}{|c|c|c|c|}
\hline$J_{g}^{P g}$ & This work $[\mathrm{Gev}]$ & $J^{P C}$ & Lattice $[14][\mathrm{Gev}]$ \\
\hline $1^{-}$ & 4.886 & $0^{-+}, 1^{-+}, 2^{-+},[7$ more $]$ & $4.291(48), 4.327(36), 4.376(24),[?]$ \\
\hline $1^{+}$ & 4.692 & $0^{+-}, 1^{+-}, 2^{++},[7$ more $]$ & $4.521(54), 4.521(48), 4.508(48),[?]$ \\
\hline $2^{-}$ & 5.221 & $1^{-+},[11$ more $]$ & $4.696(103),[?]$ \\
\hline $2^{+}$ & 5.276 & $2^{+-},[11$ more $]$ & $4.733(42),[?]$ \\
\hline
\end{tabular}

tional. This needs to be improved in particular by the effects of vortex field configurations, wildly believed to be revenant for confinement. The framework however seem to be well suited for further investigations of quarkonium and hybrid structure, including mixing and transitions.

\section{ACKNOWLEDGMENT}

We would like to thank J. Dudek for discussions of the lattice results and $\mathrm{H}$. Reinhardt for continuing discussions of the Coulomb gauge QCD. This work was supported in part by the US Department of Energy grant under contract DE-FG0287ER40365. APS also thanks the I.N.F.N and University of Genova for hospitality during preparation of this work.

\section{APPENDIX A: HAMILTONIAN MATRIX ELEMENTS}

The matrix elements of $V_{Q \bar{Q} g}^{2}$ and $V_{Q \bar{Q} g}^{3}$ in Eq. (32) are computed using gaussian wave functionals for the $Q \bar{Q}$ radial wave function.

$$
\begin{aligned}
& V_{Q \bar{Q} g}^{2,3}\left(k, k^{\prime}\right) \\
= & \int \frac{q^{2} d q}{(2 \pi)^{3}} \frac{q^{\prime 2} d q^{\prime}}{(2 \pi)^{3}} \Psi_{Q}^{L_{q}}(q) V_{Q \bar{Q} g}^{2,3}\left(k, q, \alpha ; k^{\prime}, q^{\prime}, \alpha^{\prime}\right) \Psi_{Q}^{L_{q}}\left(q^{\prime}\right)
\end{aligned}
$$

In particular for the $S$-wave, given by Eq. (34) we find

$$
\begin{aligned}
V_{Q \bar{Q} g}^{2}\left(k, k^{\prime}\right) & =\delta_{J_{g}^{\prime}, J_{g}} N_{c}\left(\sqrt{\frac{\omega(k)}{\omega\left(k^{\prime}\right)}}+\sqrt{\frac{\omega\left(k^{\prime}\right)}{\omega(k)}}\right) \sum_{l} \frac{1}{2}\left[1+P(-1)^{l}\right]\left\langle J_{g} 1 ; 1-1 \mid l 0\right\rangle^{2} \\
& \times(2 \pi) \int_{-1}^{1} d \hat{\mathbf{k}} \cdot \hat{\mathbf{k}}^{\prime} P_{l}\left(\hat{\mathbf{k}} \cdot \hat{\mathbf{k}}^{\prime}\right) \widetilde{V}_{C L}\left(\left|\mathbf{k}-\mathbf{k}^{\prime}\right|\right) e^{-\frac{\left|\mathbf{k}-\mathbf{k}^{\prime}\right|^{2}}{16 \alpha_{Q}^{2}}} .
\end{aligned}
$$

and

$$
\begin{aligned}
V_{Q \bar{Q} g}^{3}\left(k, k^{\prime}\right) & =-\frac{N_{c}^{2}}{8} \int d^{2} \widehat{\mathbf{k}} d^{2} \widehat{\mathbf{k}^{\prime}} \frac{1}{\sqrt{\omega(k) \omega\left(k^{\prime}\right)}} \sum_{m_{1}, m_{2}, \sigma, \sigma^{\prime}} \chi_{m_{1}, m_{2}, \sigma}^{* J M P C}(\hat{\mathbf{k}}, \alpha) \chi_{m_{1}, m_{2}, \sigma^{\prime}}^{J^{\prime} M^{\prime} P^{\prime} C^{\prime}}\left(\hat{\mathbf{k}^{\prime}}, \alpha^{\prime}\right) \\
& \times(4 \pi) \int \frac{d^{3} p}{(2 \pi)^{3}} K^{(2)}\left(\left|\mathbf{k}^{\prime}+\mathbf{p}\right|,|\mathbf{k}+\mathbf{p}|,|\mathbf{p}|\right)\left[2 e^{-\frac{\left|2 \mathbf{p}+\mathbf{k}+\mathbf{k}^{\prime}\right|^{2}}{16 \alpha_{Q}^{2}}}+e^{-\frac{\left|\mathbf{k}-\mathbf{k}^{\prime}\right|^{2}}{16 \alpha_{Q}^{2}}}\right] \mathbf{p} \cdot \epsilon_{-k^{\prime}, \sigma^{\prime}} \mathbf{p} \cdot \epsilon_{-k, \sigma}^{*}
\end{aligned}
$$

where $[29], \quad K^{(2)}(k, p, q)=\widetilde{V}_{C L}(k) \widetilde{V}_{C}(p) \widetilde{V}_{C}(q)+$ parity and charge conjugation satisfying $P=(-1)^{l}$ i.e. $\widetilde{V}_{C}(k) \widetilde{V}_{C L}(p) \widetilde{V}_{C}(q)+\widetilde{V}_{C}(k) \widetilde{V}_{C}(p) \widetilde{V}_{C L}(q)$. With hybrid 
the possible values of $l$ are constrained by parity of a state, $C=(-1)^{S+1}$ where is $S=0,1$ is the spin of the
$Q \bar{Q}$ pair. To order $O(1 / m)$ the other two interactions in Eq. (8) give

$$
\begin{aligned}
V_{A^{2}}^{S}\left(k, k^{\prime}\right) & =\frac{1}{4 m}\left(C_{F}-\frac{1}{2 N_{c}}\right) \frac{1}{\sqrt{\omega_{k} \omega_{k^{\prime}}}} \sum_{l}\left[1+P(-1)^{l}\right]\left\langle J_{g} 1 ; 1-1 \mid l 0\right\rangle^{2} \\
& \times \int d \hat{\mathbf{k}} \cdot \hat{\mathbf{k}}^{\prime} P_{l}\left(\hat{\mathbf{k}} \cdot \hat{\mathbf{k}}^{\prime}\right)(4 \pi)^{2} \alpha\left(\left|\mathbf{k}-\mathbf{k}^{\prime}\right|\right) e^{-\frac{\left|\mathbf{k}-\mathbf{k}^{\prime}\right|^{2}}{16 \alpha_{Q}^{2}}} .
\end{aligned}
$$

for spin-idependent contact interaction and,

$$
\begin{aligned}
V_{\sigma B}^{S}\left(k, k^{\prime}\right) & =\frac{3 N_{c}}{4 m} \frac{1}{\sqrt{\omega_{k} \omega_{k^{\prime}}}} \sum_{l}\left[1+P(-1)^{l}\right](-1)^{1+l+J}(2 S+1)(2 l+1)\left\langle 11 ; l 0 \mid J_{g} 1\right\rangle^{2} \\
& \times\left\{\begin{array}{ccc}
S & \frac{1}{2} & \frac{1}{2} \\
\frac{1}{2} & S & 1
\end{array}\right\}\left\{\begin{array}{ccc}
1 & J_{g} & J_{g} \\
l & 1 & 1
\end{array}\right\}\left\{\begin{array}{ccc}
1 & J_{g} & J_{g} \\
J & S & S
\end{array}\right\} \int d \hat{\mathbf{k}} \cdot \hat{\mathbf{k}}^{\prime} P_{l}\left(\hat{\mathbf{k}} \cdot \hat{\mathbf{k}}^{\prime}\right)(4 \pi)^{2} \alpha\left(\left|\mathbf{k}-\mathbf{k}^{\prime}\right|\right) e^{-\frac{\left|\mathbf{k}-\mathbf{k}^{\prime}\right|^{2}}{16 \alpha_{Q}^{2}}}
\end{aligned}
$$

for spin-dependent contact interaction. Here

$$
\alpha(p)=\frac{4 \pi Z}{\beta^{\frac{3}{2}} \ln ^{\frac{3}{2}}\left(\frac{p^{2}}{\Lambda_{Q C D}^{2}}+c\right)}
$$

with $Z=5.94, c=40.68, \Lambda_{Q C D}=0.25 \mathrm{GeV}$ arising from the fit to the Coulomb part of the QCD potential [29].
[1] C. J. Morningstar and M. J. Peardon, Phys. Rev. D 60, 034509 (1999) [arXiv:hep-lat/9901004].

[2] M. Foster and C. Michael [UKQCD Collaboration], Phys. Rev. D 59, 094509 (1999) [arXiv:hep-lat/9811010].

[3] G. S. Bali and A. Pineda, Phys. Rev. D 69, 094001 (2004) [arXiv:hep-ph/0310130].

[4] D. Horn and J. Mandula, Phys. Rev. D 17, 898 (1978).

[5] N. Isgur and J. E. Paton, Phys. Rev. D 31, 2910 (1985).

[6] Yu. A. Simonov, Nucl. Phys. B 592, 350 (2001) [arXiv:hep-ph/0003114].

[7] A. Szczepaniak, E. S. Swanson, C. R. Ji and S. R. Cotanch, Phys. Rev. Lett. 76, 2011 (1996)

[8] F. J. Llanes-Estrada and S. R. Cotanch, Phys. Lett. B 504, 15 (2001) [arXiv:hep-ph/0008337].

[9] I. J. General, S. R. Cotanch and F. J. Llanes-Estrada, Eur. Phys. J. C 51, 347 (2007) [arXiv:hep-ph/0609115].

[10] F. Buisseret and C. Semay, Phys. Rev. D 74, 114018 (2006) [arXiv:hep-ph/0610132].

[11] F. Brau and C. Semay, Phys. Rev. D 70, 014017 (2004) [arXiv:hep-ph/0412173].

[12] K. J. Juge, J. Kuti and C. Morningstar, Phys. Rev. Lett. 90, 161601 (2003) [arXiv:hep-lat/0207004].

[13] K. J. Juge, J. Kuti and C. J. Morningstar, Phys. Rev. Lett. 82, 4400 (1999) [arXiv:hep-ph/9902336].

[14] J. J. Dudek, R. G. Edwards, N. Mathur and D. G. Richards, Phys. Rev. D 77, 034501 (2008) [arXiv:0707.4162 [hep-lat]].
[15] E. Eichten and F. Feinberg, Phys. Rev. D 23, 2724 (1981).

[16] Yu. A. Simonov, Nucl. Phys. B 324, 67 (1989).

[17] A. Barchielli, N. Brambilla and G. M. Prosperi, Nuovo Cim. A 103, 59 (1990).

[18] F. L. Feinberg, Phys. Rev. D 17, 2659 (1978).

[19] J. Greensite and S. Olejnik, Phys. Rev. D 67, 094503 (2003) [arXiv:hep-lat/0302018].

[20] A. P. Szczepaniak and P. Krupinski, Phys. Rev. D 73, 116002 (2006) [arXiv:hep-ph/0604098].

[21] A. P. Szczepaniak and P. Krupinski, Phys. Rev. D 73, 034022 (2006) [arXiv:hep-ph/0511083].

[22] A. P. Szczepaniak and E. S. Swanson, Phys. Rev. D 65, 025012 (2002) [arXiv:hep-ph/0107078].

[23] A. P. Szczepaniak, Phys. Rev. D 69, 074031 (2004) [arXiv:hep-ph/0306030].

[24] C. Feuchter and H. Reinhardt, Phys. Rev. D 70, 105021 (2004) [arXiv:hep-th/0408236].

[25] H. Reinhardt and C. Feuchter, Phys. Rev. D 71, 105002 (2005) [arXiv:hep-th/0408237].

[26] W. Schleifenbaum, M. Leder and H. Reinhardt, Phys. Rev. D 73, 125019 (2006) [arXiv:hep-th/0605115].

[27] D. Epple, H. Reinhardt and W. Schleifenbaum, Phys. Rev. D 75, 045011 (2007) [arXiv:hep-th/0612241].

[28] D. Epple, H. Reinhardt, W. Schleifenbaum and A. P. Szczepaniak,

[29] P. Guo, A. P. Szczepaniak, G. Galata, A. Vassallo and 
E. Santopinto, arXiv:0707.3156 [hep-ph].

[30] J. J. Dudek, In the Proceedings of International Workshop on Charm Physics (Charm 2007), Ithaca, New
York, 5-8 Aug 2007, pp 21 [arXiv:0711.1600 [hep-ph]]. 